\title{
An Introduction to IAU 2009 Resolution B5
}

\author{
Malcolm G. Smith \\ Cerro Tololo Interamerican Observatory, \\ Casilla 603, La Serena, Chile \\ email: msmith@ctio.noao.edu
}

\begin{abstract}
For the purposes of the discussion of IAU follow up on Resolution B5, adopted at the GA in Rio de Janiero in 2009, reference is made here to the resolution itself and the background which led to its proposal and adoption by the IAU.
\end{abstract}

Keywords. Light pollution, IAU resolution

IAU 2009 Resolution B5 was distributed to participants at the beginning of the discussion session here in Beijing. The orginal resolution was initially drafted by Pedro Russo and Connie Walker. It can be found at

http://www.iau.org/static/resolutions/IAU2009_English.pdf

It refers to the Starlight Initiative http://www.starlight2007.net/ and recognises a series of principles raised in a declaration approved by that Initiative in 2007. It recognises the competent bodies within the IAU to work on such matters - namely Commission 50 and its WG for Controlling Light Pollution, as well as the IYA Cornerstone Project "Dark Skies Awareness". It then lists a series of considerations concerning the adverse effects of light pollution and urges IAU members to raise public awareness of the aims of the Starlight Initiative, "in particular the educational, scientific, cultural, health and recreational importance of preserving access to an unpolluted night sky for all humankind".

Returning focus to the scientific needs of professional astronomy, Resolution B5 resolves that "Protection of the astronomical quality of areas suitable for scientific observation of the Universe should be taken into account when developing and evaluating national and international scientific policies, with due regard to local cultural and natural values."

Following on from the first meeting of the Starlight Initiative, many aspects of Resolution B5 have been taken up in collaboration with IAU Commission 41, History of Astronomy, in particular with its Astronomy and World Heritage Working Group, under the leadership of Clive Ruggles. On 24th August 2012, (following planning carried out a couple of months earlier at a meeting in New Zealand) a new UNESCO-IAU online Portal to the Heritage of Astronomy was launched at the General Assembly. As stated in the press release, "The site, which resides at www.astronomicalheritage.net, is a dynamic, publically accessible database, discusssion forum and document repository on astronomical heritage sites throughout the world, even if they are not on UNESCO's World Heritage List."

In addition to considering ancient heritage sites such as Stonehenge, the concept of starlit skies is advanced as a Scientific and Cultural heritage for future generations which will be lost if not protected now. Included among such sites are "Windows to the Universe", one of which (Northern Chile) is discussed here in the subsession on "Light Pollution Legislation and Protecting Observatory Sites". These "Windows" are groundbased sites in the northern and southern hemisphere considered particularly suitable for professional, international, optical observatories. 